BULL. AUSTRAL. MATH. SOC.

VOL. 9 (1973), 363-366.

\title{
On groups admitting a noncyclic abelian automorphism group
}

\section{J.N. Ward}

It is shown that a condition of Kurzwell concerning fixed-points of certain operators on a finite group $G$ is sufficient to ensure that $G$ is soluble. The result generalizes those of Martineau on elementary abelian fixed-point-free operator groups.

Suppose that $V$ is an abelian group of operators on the finite group $G$. Kurzweil [3] studied soluble groups which satisfy the condition:

(*) for each prime divisor $q$ of $\left|C_{G}(V)\right|$ and for each $v \in V^{\#}$ the $q$-elements of $C_{G}(V)$ centralize the $q^{\prime}$-elements of $C_{G}(v)$.

The purpose of this note is to prove the following theorem.

THEOREM. Suppose that $V$ is a noncyclic elementary abelion $r$-group of operators on the finite $r^{\prime}$-group $G$ for some prime $r$. Assume that the condition (*) is satisfied. Then $G$ is soluble. If $|V|=r^{n}$ then $G=F_{n}(G)$.

Here $E_{n}(G)$ denotes the $n$-th term of the upper Fitting series of $G$. The other notation is standard and, in any case, agrees with that of [2].

It is hardly necessary to remark that the condition (*) is satisfied whenever $C_{G}(V)=1$. Thus the theorem directly extends the theorems of Martineau ([4] and [5]) on groups with elementary abelian fixed-point-free

Received 21 June 1973. 
operator groups.

Another special case of interest is that in which ${ }_{C}(v)$ is nilpotent for each $v \in V^{\#}$. In this case we conclude that $G$ is soluble. In fact more can be said and the relevant results appear in [7] and [8] for the cases $m(V)=2$ and $m(V) \geq 3$ respectively.

The theorem also has an application in the study of rank 3 signalizer functors on finite groups.

The proof of the theorem which is given below reduces the proof of solubility to the special case which was considered by Martineau. I am grateful to Mr $P$. Rowley for suggesting this reduction.

Proof. The first step is to show that a group which satisfies the hypothesis of the theorem is soluble. We use the notation of the statement of the theorem and proceed by induction on $|G|$. Clearly we may assume that $G$ is characteristically simple.

By the corollary to the main theorem of [5] we may suppose that $C_{G}(V) \neq 1$. Choose in $C_{G}(V)$ an element $x$ which has prime order - say $x$ has order $q$. Let $p$ denote any prime divisor of $|G|$ different from $q$ and let $P$ denote a V-invariant Sylow $p$-subgroup of $G$. (Such a subgroup $P$ exists by Theorem 6.2.2 of [2]). Since $V$ is noncyclic it follows that

$$
P=\left\langle c_{P}(v) \mid v \in v^{\#}\right\rangle
$$

But by our hypothesis (*) we know that $C_{P}(v) \leq C_{G}(x)$. Hence $P$ is contained in $C_{G}(x)$ so that the number of conjugates of $x$ is prime to $p$. From our arbitrary choice of $p$, we deduce that the number of conjugates of $x$ in $G$ is a power of $q$.

It now follows from Burnside's Lemma ([2], Lemma 4.3.2) that $G$ is not a nonabelian simple group. A slight extension of this lemma yields that $G$ must be elementary abelian and hence soluble. This completes the proof of the solubility of $G$.

We now assume that $|V|=r^{n}$ and prove that $G=F_{n}(G)$. Again we 
proceed by induction on $|G|$. By Lemma 2, p. 482 of [6], $F(G)$ is the unique minimal $V$-invariant normal subgroup of $G$. Hence $F(G)$ is an elementary abelian $p$-group for some prime $p$ and $F_{2}(G) / F(G)$ has order prime to $p$.

Now the above proof shows that if $x \in C_{G}(V)$ and $x$ has prime order $q$, which is distinct from $p$, then $x \in C_{G}(F(G))$. By a well known property of soluble groups $x$ is contained in $F(G)$. But this contradicts the choice of $x$. Hence $C_{G}(V)$ is a p-group.

The same argument now shows that $C_{G}(V)$ centralizes $F_{2}(G) / F(G)$. Hence $C_{G}(V) \leq F_{2}(G)$. Since $F(G)$ is the Sylow p-subgroup of $F_{2}(G)$ we may conclude that $C_{G}(V) \leq Z\left(F_{2}(G)\right)$.

On the other hand, our characterization of $F(G)$ as the unique minimal normal $V$-invariant subgroup of $G$ forces us to conclude that $Z\left(F_{2}(G)\right)=1$. Hence $C_{G}(V)=1$. We may now apply the main theorem of [1], to obtain the required conclusion.

\section{References}

[1] T.R. Berger, "Nilpotent fixed-point-free automorphism groups", (to appear).

[2] Daniel Gorenstein, Finite groups (Harper and Row, New York, Evanston, London, 1968).

[3] Hans Kurzweil, "Auflösbare Gruppen, die eine abelsche Automorphismengruppe gestatten, deren Fixpunktgruppe nilpotent ist", J. Algebra 10 (1968), 92-101.

[4] R. Patrick Martineau, "Solubility of groups admitting a fixed-pointfree automorphism group of type $(p, p)$ ", Math. Z. 124 (1972), $67-72$.

[5] R. Patrick Martineau, "Elementary abelian fixed point free automorphism groups", Quart. J. Math. Oxford (2) 23 (1972), 205-212. 
[6] J.N. Ward, "Involutory automorphisms of groups of odd order", $J$. Austral. Math. Soc. 6 (1966), 480-494.

[7] J.N. Ward, "Automorphisms of finite groups and their fixed-point groups", J. Austral. Math. Soc. 9 (1969), 467-477.

[8] J.N. Ward, "On finite groups admitting automorphisms with nilpotent fixed-point group", Bull. Austral. Math. Soc. 5 (1971), 281-282.

Department of Pure Mathematics, University of Sydney,

Sydney, New South wales. 